\title{
Image fusion algorithm for differential phase contrast imaging
}

\author{
Ewald Roessl ${ }^{a}$, Thomas Koehler ${ }^{a}$, Udo van Stevendaal ${ }^{a}$, Gerhard Martens ${ }^{a}$, Nik Hauser ${ }^{b}$, \\ Zhentian Wang ${ }^{c}$ and Marco Stampanoni ${ }^{c, d}$ \\ ${ }^{a}$ Philips Technologie GmbH, Innovative Technologies, Research Laboratories Hamburg, \\ Germany \\ ${ }^{b}$ Department of Gynecology and Obstetrics, Interdisciplinary Breast Center Baden, \\ Kantonsspital Baden, 5404 Baden, Switzerland \\ ${ }^{c}$ Swiss Light Source, Paul Scherrer Institut, 5232 Villigen, Switzerland \\ ${ }^{d}$ Institute for Biomedical Engineering, University and ETH Zürich, 8092 Zürich, Switzerland
}

\begin{abstract}
Differential phase-contrast imaging in the x-ray domain provides three physically complementary signals: ${ }^{1,2}$ the attenuation, the differential phase-contrast, related to the refractive index, and the dark-field signal, strongly influenced by the total amount of radiation scattered into very small angles. In medical applications, it is of the utmost importance to present to the radiologist all clinically relevant information in as compact a way as possible. Hence, the need arises for a method to combine two or more of the above mentioned signals into one image containing all information relevant for diagnosis. We present an image composition algorithm that fuses the attenuation image and the differential phase contrast image into a composite, final image based on the assumption that the real and imaginary part of the complex refractive index of the sample can be related by a constant scaling factor. The merging is performed in such a way that the composite image is characterized by minimal noise-power at each frequency component.
\end{abstract}

Keywords: differential phase contrast imaging, Talbot-Lau interferometry, image composition, mammography

\section{INTRODUCTION}

The progress over the last couple of years in the field of x-ray differential phase contrast imaging using diffraction gratings has been tremendous ${ }^{1-4}$ both in terms of the development of novel methods and in terms of the fabrication of gratings for increasingly high design energies. Of particular importance to potential applications in medical imaging was the development of a technique that enabled the use of conventional x-ray tubes as sources of radiation by the addition of a partially attenuating source grating that generates partially coherent x-rays. ${ }^{1}$ Moreover, the possibility to quantify the amount of small angle scattering by the sample at structures well below the physical resolution of the imaging system ${ }^{2,5}$ increased the interest of many researchers in the medical domain even further. Today, x-ray gratings are readily available for design energies of up to 30-40 keV fully covering the energy domain of x-ray mammography and approaching even the average energies employed in human computed tomography.

When the phase information is obtained via phase-retrieval from a complete phase stepping scan (typically about 8 exposures with any of the three gratings displaced perpendicular to the x-rays and the trench structures during each phase step), three pieces of information can be derived from it: the mean attenuation by the sample, computed as the mean intensity over the phase-stepping cycle (often transformed into a line-integral over the linear attenuation by normalizing to the intensity without sample and taking the logarithm), the differential phase contrast image as the phase of the observed intensity modulation and the "de-coherence" or scattering contribution, as the amplitude of the intensity modulation observed during the phase stepping relative to the mean signal.

From a perspective of diagnostic imaging the question arises whether the differential phase contrast image and the scattering image contain additional, clinically relevant information and if so, what would be the best

Further author information: (Send correspondence to E.R.)

E.R.: E-mail: ewald.roessl@philips.com, Telephone: +49 (0)40 50782965

Medical Imaging 2012: Physics of Medical Imaging, edited by Norbert J. Pelc, Robert M. Nishikawa, Bruce R. Whiting, Proc. of SPIE Vol. 8313, 831354 - @ 2012 SPIE · CCC code: 1605-7422/12/\$18 · doi: 10.1117/12.911255 
way to present this information to the clinician in a most compact and useful way avoiding the tedious task of reading 3 images in parallel. The aim of this paper is to provide a possible answer to the above question by introducing an image fusion algorithm that combines the attenuation and the differential phase information in a way optimized for noise.

The present paper is structured in the following way: in Section 2, the algorithm is described together with the most important underlying assumptions. In the following Section 3 we present results of the application of the algorithm in two concrete examples. The first example is a scan of a cable strap with the typical saw-tooth structures corresponding to an almost ideal phase object featuring areas with constant phase gradient. We compare the attenuation image to the result of our image fusion algorithm and measure the decrease in image noise and discuss the implications. As a second example we apply the image fusion algorithm to data recently acquired on human mastectomy samples ${ }^{6}$ and discuss the improvements in image quality with respect to the attenuation image. We finally conclude by discussing current shortcomings of the present algorithm and possible extension in Section 4.

\section{METHODS}

\subsection{Algorithm}

By the very nature of the acquisition of a complete phase stepping cycle, the three data sets derived after phase retrieval are automatically registered in the spatial domain. The idea to the present algorithm resulted from the observation that regularized integrated images of the differential phase image have a strong similarity to the images showing attenuation apart from the very different noise power spectra introduced by the integration step. Whereas the attenuation image depicts line-integrals of the linear attenuation coefficient of the sample, the integrated, differential-phase signal depicts line-integrals of the refractive index decrement. The attenuation is dominated either by the photo-electric effect at low x-ray energies with a very strong dependence on energy or by the Compton effect at higher energies. The refractive index (and its decrement) similar to the Compton-effect is essentially proportional to the local electron density within the sample while the photo-electric effect shows a strong additional dependence (by the third power) on the effective atomic number of the sample, see, e.g. ${ }^{7}$

The algorithm we derive in the following neglects the latter fact and is based on the assumption that, at least approximately, the linear attenuation coefficient $\mu$ and the refractive index decrement $\delta$ of the sample at a given effective energy of the setup are related throughout the image by a simple scaling transformation of the type:

$$
\delta(x, y, z)=c \mu(x, y, z) .
$$

The validity of this approximation depends on the relative importance of the Compton-effect as compared to the photo-electric effect and on the magnitude of the variations in effective atomic number in the sample.* From the above assumption it immediately follows that the Fourier transforms of the measured quantities $f(x, y)$ and $g(x, y)$, denoting the line-integrals of the attenuation and the gradient of the refractive index decrement, respectively,

$$
\begin{aligned}
& f(x, y) \quad=\int_{\mathcal{C}} \mu(x, y, z) d z \\
& g(x, y)=\int_{\mathcal{C}} \frac{\partial}{\partial x} \delta(x, y, z) d z
\end{aligned}
$$

are related via the following equation:

$$
G\left(k_{x}, y\right)=-2 \pi i k_{x} c F\left(k_{x}, y\right)
$$

where we assume a parallel beam geometry for simplicity with $\mathrm{z}$ being the direction of propagation of the $\mathrm{x}$ rays. $F\left(k_{x}, y\right)$ and $G\left(k_{x}, y\right)$ denote the 1-dimensional Fourier transform of the image data along the direction

\footnotetext{
*In the case of mammography it will certainly be violated for micro-calcifications where the effective atomic number will dominate the attenuation through the photo-electric effect and locally the ratio of attenuation to refractive index decrement will deviate strongly from the corresponding ratio in the background.
} 
perpendicular to the x-ray gratings. Using the fact that the noise in $f(x, y)$ and $g(x, y)$ after phase retrieval is practically uncorrelated (see, e.g., ${ }^{8}$ p. 56 or $^{9}$ ), we can derive frequency dependent filter functions $\omega_{1}\left(k_{x}, c\right)$ and $\omega_{2}\left(k_{x}, c\right)$ for a noise-optimal combination $h(x, y)$ of the two images images $f(x, y)$ and $g(x, y)$ in Fourier space according to the following equation:

$$
H\left(k_{x}, y\right)=\omega_{1}\left(k_{x}, c\right) F\left(k_{x}, y\right)+\omega_{2}\left(k_{x}, c\right) \frac{1}{-2 \pi i k_{x} c} G\left(k_{x}, y\right),
$$

where $\omega_{1}\left(k_{x}, c\right)$ and $\omega_{2}\left(k_{x}, c\right)$ are given by:

$$
\omega_{1}\left(k_{x}, c\right)=\frac{1}{1+\left(\frac{k_{x}}{k_{0}}\right)^{2}} \quad \text { and } \quad \omega_{2}\left(k_{x}, c\right)=\frac{\left(\frac{k_{x}}{k_{0}}\right)^{2}}{1+\left(\frac{k_{x}}{k_{0}}\right)^{2}}
$$

and where the constant "cut-off" frequency $k_{0}$ is defined by

$$
k_{0}=\frac{1}{2 \pi c} \sqrt{\frac{\sigma_{g}^{2}}{\sigma_{f}^{2}}}
$$

The choice expressed in Eq. (6) minimizes the noise in $H\left(k_{x}, y\right)$, expressed by Eq. (5) at each frequency component $k_{x} . \sigma_{f}^{2}$ and $\sigma_{g}^{2}$ denote typical values for the noise variance in the images $f(x, y)$ and $g(x, y)^{\dagger}$. Note that at each frequency $k_{x}$ the filter functions were required to add up to one, which we imposed because the signal was required to be left unchanged by the fusion algorithm, only the noise should be reduced at each frequency component. Moreover, the zero in the filter function $\omega_{2}\left(k_{x}, c\right)$ at $k_{x}=0$ correctly accounts for the intuitive low significance of the differential measurement at low frequencies and guarantees that the Fourier coefficient of the fused image at that point remains finite and determined by the attenuation measurement only. It is instructive to see how the requirement for lowest noise at each frequency component translates into the characteristic change in dominance of the two weighting functions at high, and low frequencies, respectively.

\subsection{Experimental setups and phantoms}

The algorithm was applied to two different example cases acquired at two different differential phase contrast setups. The scan data of the cable strap test phantom with a sawtooth pattern was acquired at a test-setup built at Philips Research in Hamburg. Source-, phase-, and analyzer gratings were purchased from Centre Suisse d'Electronique et Microtechnique, SA (CSEM), Switzerland and designed for an energy of $25 \mathrm{keV}$, corresponding to grating periodicities of $p_{0}=30.0 \mu \mathrm{m}, p_{1}=5.45 \mu \mathrm{m}$ and $p_{2}=3.00 \mu \mathrm{m}$, respectively. The source-grating to phasegrating distance and the phase-grating to analyzer grating distance were $825 \mathrm{~mm}$ and $82.5 \mathrm{~mm}$, respectively (1st fractional Talbot distance) for a phase-grating of phase-shift $\pi$. As x-ray detector we used a Radicon Shad-oBox $^{\mathrm{TM}}$ with a pixel size of $50 \mu \mathrm{m} \times 50 \mu \mathrm{m}$, as x-ray tube a Comet MXR fixed anode tungsten target tube with a focus spot size of about $300-400 \mu \mathrm{m}$.

The differential phase-contrast scan of native human breast mastectomy samples from the Kantonsspital in Baden, Switzerland, was performed at the Paul-Scherrer-Institue (PSI), Switzerland with a setup designed and built by PSI. The details of the setup can be found in. ${ }^{6}$ In brief, the x-ray setup consisted of a Seifert ID 3000 x-ray generator and an unfiltered tungsten line focus tube. The design energy was fixed at $28 \mathrm{keV}$. As x-ray detector a Hamamatsu C9732DK flat panel CMOS detector featuring a 12 x 12-cm field of view with $50 \mu \mathrm{m} \times 50 \mu \mathrm{m}$ pixel size was used. Also here, a three grating phase-contrast setup has been used to acquire phase information by the phase-stepping approach.

\footnotetext{
${ }^{\dagger}$ Note that we also assume that the noise power spectra of $f(x, y)$ and $g(x, y)$ are identical up to a constant, so that the ratio $\frac{\sigma_{g}^{2}}{\sigma_{f}^{2}}$ is independent of the frequency $k_{x}$. In the absence of detector crosstalk, one expects white Gaussian noise for both, $f(x, y)$ and $g(x, y)$.
} 


\section{RESULTS}

The results of the scan of the cable strap are shown in Fig. 1 below. Part (a) of Fig. 1 shows the integrated differential phase contrast signal and illustrates the amplification of low frequency noise during the Fourier-based integration. Note that the zero-frequency component in this part of the figure was taken from the corresponding component from the attenuation image shown in part (b) as this information is unavailable from the differential phase contrast signal. The image fusion results according to the proposed algorithm are shown in part (c) of Fig. 1.

Noise measurements were performed in a small region-of-interest in the flat part of the cable strap and demonstrate the benefits of the algorithm and the corresponding improvement in image quality. The reduced noise level in the fused image shown in Fig. 1 part (c) can also be appreciated visibly when compared to the noise in part (b). The ratio of the signal-to-noise-ratios between the fused image and the attenuation component was measured to be 1.8. We note that, after compensating for the effect that about $50 \%$ of the radiation that has passed the object does not directly contribute to the attenuation contrast, i.e., is absorbed in the analyzer grating, the fused image still outperforms the attenuation image. This insight does have consequences for mammography, where radiation dose to the patient is of the highest importance.

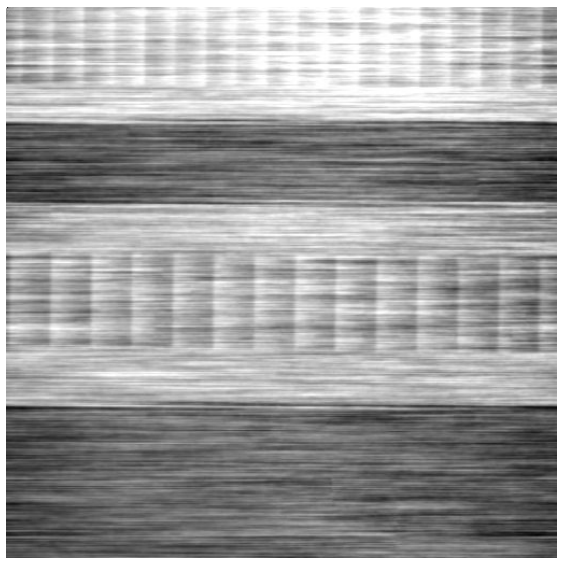

(a)

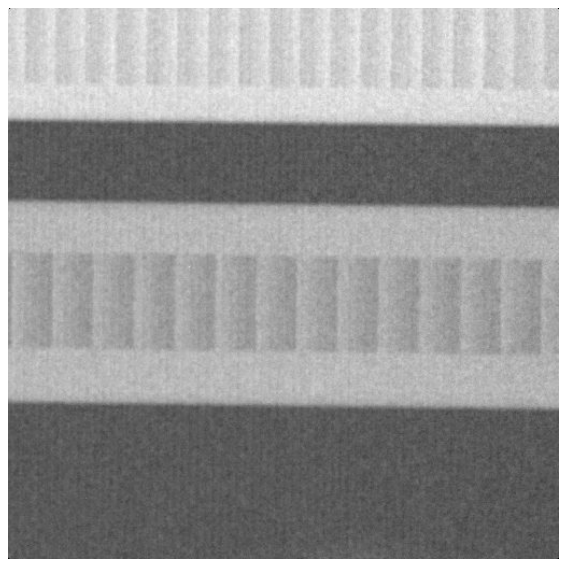

(b)

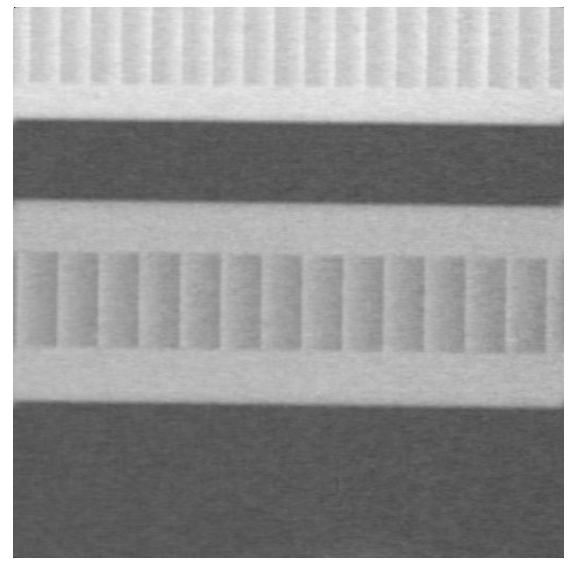

(c)

Figure 1. Integrated phase image (unregularized) (a), attenuation image (b) and fused image (c) as obtained with the proposed algorithm. All images are displayed on a linear grey-scale ranging from 0 (black) to 0.03 (white) and represent the line-integrals of the linear attenuation coefficient (either directly as in the case of (b) or indirectly as in the case of (a) and (c)) based on the assumption of proportionality between $\mu$ and $\delta$.

The results of the scan of a human mastectomy sample from a female patient with an invasive, ductal breast carcinoma, aged 70 years (case 2 of $^{6}$ ) are shown in Fig. 2. Part (a) of Fig. 2 shows the attenuation image, part (b) the differential phase-contrast image, whereas part (c) shows the result of the image fusion algorithm. The overal impression is an improved visualization of fine (high-frequency) details as well as interfaces between different tissue types, attributed to the lower noise power in the differential phase contrast image at high frequencies. Note that the images show only a small region of the total scan field of view.

\section{CONCLUSIONS}

We presented an algorithm to combine data from differential phase contrast projection imaging devices in a noise optimal way under the assumption of a simple scaling law between the attenuation coefficient $\mu$ and refractive index decrement $\delta$. This assumption is expected to be well satisfied for biological tissue like breast tissue where the local changes in the effective atomic number of the tissue are relatively small. Very importantly, we found that the achieved increase in SNR in the case of the cable strap test-phantom by about a factor of 1.8 is already above the required increase for compensating for the loss of usable radiation in the analyzer gratings, which would be a factor of $\sqrt{2}$. This result can also be interpreted in terms of the possibility to reduce the dose by means of phase-contrast imaging while maintaining SNR without sacrificing spatial resolution. This result will 


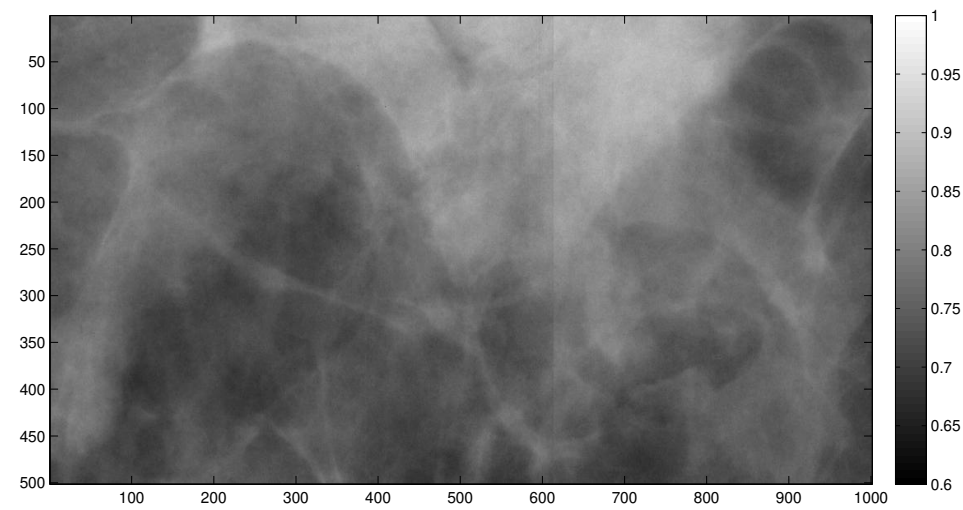

(a)

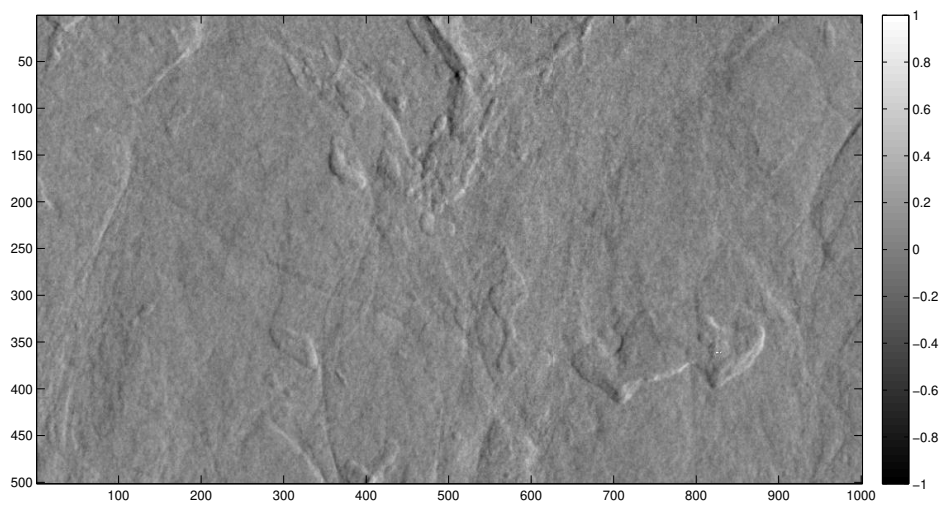

(b)

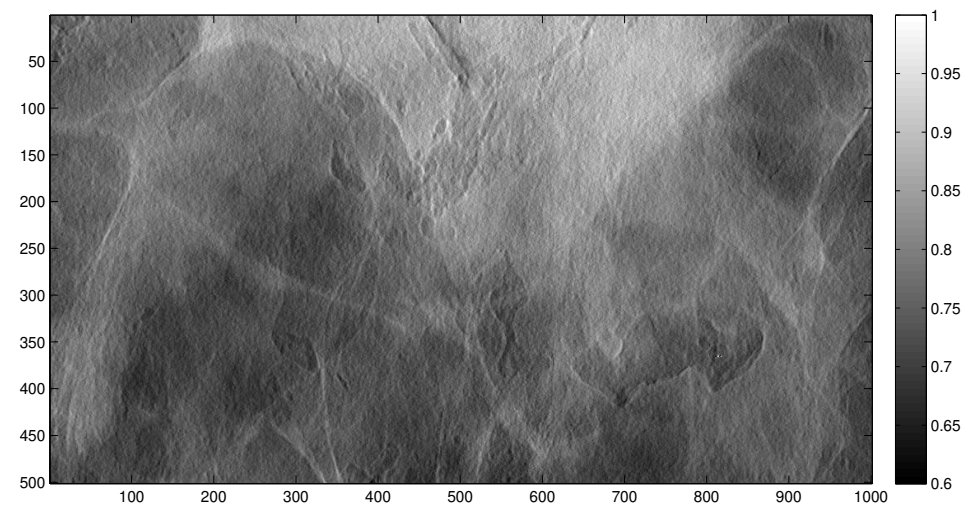

(c)

Figure 2. Attenuation image (a), differential phase contrast image (b) and fused image (c) as obtained with the proposed algorithm applied to data from a scan of a native, human breast tissue sample. The images (a) and (c) are displayed on a linear grey-scale ranging from 0.6 (black) to 1.0 (white) and represent the line-integrals of the linear attenuation coefficient. The image (b) shows the relative phase of the intensity modulation over the entire detector from -1 rad to $1 \mathrm{rad}$. 
be investgated in more detail in the future also in terms of the influence of the object thickness. Our results seem to indicate, however, that already the fusion of the attenuation image and the differential phase contrast image holds the potential to compensate for the problems related to dose utility in this technique due to typically highly attenuating analyzer gratings. The information of the dark-field image ${ }^{2}$ has not been taken into account yet and might further be a useful ingredient for more complete fusion algorithms.

We identified another possible extension of our algorithm by the observation of a shortcoming related to micro-calcifications. Our scanning results for the mastectomy sample indicate that the proposed fusion algorithm improves the visibility of high-frequency details and tissue boundaries in the breast. However, we also observed in other cases that micro-calcifications which may be very important indirect signs in the development of breast cancer do remain unenhanced by our algorithm or even get slightly suppressed. This result, we explain by the fact that the optimal proportionality constant $c$ between $\mu$ and $\delta$ varies significantly for soft-tissue und microcalcifications because of the strong changes of $\mu$ through the high effective atomic number of the calcium. In future work we would also like to develop an extension of the present algorithms that can overcome the problems mentioned in relation to micro-calcifications.

\section{ACKNOWLEDGMENTS}

We would like to thank Nataly Wieberneit, Ingrid Schulze-Wenck and Hanns-Ingo Maack from Philips Healthcare, Women's Health for numerous dicussions on the processing and presentation of the images and on the valuable feedback during the stages of the development of the image fusion algorithm.

\section{REFERENCES}

[1] Pfeiffer, F., Weitkamp, T., Bunk, O., and David, C., "Phase retrieval and differential phase-contrast imaging with low-brilliance X-ray sources," Nature Phys. 2, 258 - 261 (2006).

[2] Pfeiffer, F., Bech, M., Bunk, O., Kraft, P., Eikenberry, E. F., Brönnimann, C., Grünzweig, C., and David, C., "Hard-X-ray dark-field imaging using a grating interferometer," Nature Mater. 7, 134 - 137 (2008).

[3] David, C., Nohammer, B., Solak, H. H., and Ziegler, E., "Differential x-ray phase contrast imaging using a shearing interferometer," Appl. Phys. Lett. 81(17), 3287-3289 (2002).

[4] Weitkamp, T., Diaz, A., David, C., Pfeiffer, F., Stampanoni, M., Cloetens, P., and Ziegler, E., "X-ray phase imaging with a grating interferometer," Opt Express 13, 6296-6304 (Aug 2005).

[5] Yashiro, W., Terui, Y., Kawabata, K., and Momose, A., "On the origin of visibility contrast in x-ray Talbot interferometry.," Opt Express 18, 16890-16901 (Aug 2010).

[6] Stampanoni, M., Wang, Z., Thring, T., David, C., Roessl, E., Trippel, M., Kubik-Huch, R. A., Singer, G., Hohl, M. K., and Hauser, N., "The first analysis and clinical evaluation of native breast tissue using differential phase-contrast mammography," Investigative Radiology 46, 801-806 (2011).

[7] Alvarez, R. E. and Macovski, A., "Energy-selective reconstructions in x-ray computerized tomography," Phys. Med. Biol. 21(5), 733-744 (1976).

[8] Kay, S. M., [Fundamentals of statistical signal processing, Volume 1, Estimation Theory], Prentice Hall Signal Processing Series, Prentice Hall PTR, New Jersey (1993).

[9] Weber, T., Bartl, P., Bayer, F., Durst, J., Haas, W., Michel, T., Ritter, A., and Anton, G., "Noise in x-ray grating-based phase-contrast imaging.," Med. Phys. 38, 4133-4140 (Jul 2011). 